\title{
Admissible Differential Bases in Banach Spaces
}

\author{
Evgenii I. Berezhnoj \\ Yaroslavl State University, Sovetskaya Street 14, Yaroslavl 150000, Russia \\ Correspondence should be addressed to Evgenii I. Berezhnoj; ber@uniyar.ac.ru
}

Received 27 February 2013; Accepted 2 July 2013

Academic Editor: Marco Squassina

Copyright (C) 2013 Evgenii I. Berezhnoj. This is an open access article distributed under the Creative Commons Attribution License, which permits unrestricted use, distribution, and reproduction in any medium, provided the original work is properly cited.

In the present work, a new method for constructing differential bases is presented. The bases constructed by this method allow us to distinguish symmetric spaces with different behaviour of fundamental functions at zero, as in the case of Hayes and Stokolos, and even allow us to distinguish Lorentz and Marzinkiewicz spaces or Lebesgue and Marzinkiewicz spaces whose fundamental functions are the same.

\section{Introduction}

We recall certain definitions connected with differential bases [1]. By a differential basis $B(t)$ at a point $t \in \mathbb{R}^{n}$ is meant a family of bounded measurable sets with positive measure which contain $t$ and are such that there is at least one sequence $\left\{B_{k} \in B(t)\right\}$ satisfying the condition diam $B_{k} \rightarrow 0$. A union of such families $\mathbf{B}=\left\{\bigcup B(t): t \in \mathbb{R}^{n}\right\}$ is called a differential basis in $R^{n}$. We note that the bulk of the problems and assertions in the theory of differentiation of integrals in $\mathbb{R}^{n}$ consists of testing the validity almost everywhere of some fact. Therefore, it is natural to consider differential bases which are defined, not for all points in $\mathbb{R}^{n}$, but only almost everywhere. In what follows, we will make use of these observations.

The classical examples of differential bases are the bases in $\mathbb{R}^{n}$, usually denoted by $\mathbf{B}_{s}\left(\mathbb{R}^{n}\right)(s=1,2, \ldots, n)$ and consisting of all rectangular parallelepipeds $B$ of the form

$$
B=\left\{\left(t_{1}, \ldots, t_{n}\right) \in \mathbb{R}^{n}: \alpha_{i}<t_{i}<\alpha_{i}+\gamma_{i}, i=1, \ldots, n\right\},
$$

which satisfy the condition $\gamma_{j}=\gamma_{1}$ for $j=1, \ldots, s$ and $\alpha_{i} \in \mathbb{R}$, $\gamma_{i} \in \mathbb{R}_{+}$.

A basis B made up of open sets is called a Busemann-Feller basis (BF-basis) if it follows from the conditions $B \in \mathbf{B}$ and $t \epsilon$ $B$ such that $B \in B(t)$. The significance of the introduction of BF-bases lies in the fact that questions arising in the theory of differentiation with respect to bases can be easily resolved for $\mathrm{BF}$ bases. We now define the upper and lower derivatives of the integral of a locally integrable function $f$ at a point $t \in \mathbb{R}^{n}$ with respect to a basis $\mathbf{B}$ by means of the identities

$$
\begin{aligned}
& \overline{D_{\mathbf{B}}(f, t)} \\
& \quad=\sup \left\{\varlimsup_{k \rightarrow \infty} \frac{1}{\mu\left(B_{k}\right)} \int_{B_{k}} f d s: B_{k} \in B(t), \operatorname{diam} B_{k} \longrightarrow 0\right\}, \\
& \frac{D_{\mathbf{B}}(f, t)}{\quad} \\
& \inf \left\{\frac{\lim }{k \rightarrow \infty} \frac{1}{\mu\left(B_{k}\right)} \int_{B_{k}} f d s: B_{k} \in B(t), \operatorname{diam} B_{k} \longrightarrow 0\right\} .
\end{aligned}
$$

We note that the upper and lower derivatives are variants of the functionals $\mu^{*}$ and $\mu_{*}$ which are the subject of investigation in several sections of the chapter 1 in [2].

Following [1], we say that a basis $\mathbf{B}$ differentiates the integral of $f$ if the identities

$$
\overline{D_{\mathbf{B}}(f, t)}=\underline{D_{\mathbf{B}}(f, t)}=f(t)
$$

hold almost everywhere. If $\mathbf{B}$ differentiates the integral of any function in the space $X$, then we say that the basis B differentiates the space $X$. If the basis $\mathbf{B}$ differentiates $L^{\infty}$, then it is said to be a density basis [1].

One of the fundamental problems of the theory of differentiation of integrals has the following form: given two function spaces $X, Y$ which are different in some sense, is 
it possible to distinguish these two spaces with the help of differential bases? In other words, does there exist a differential basis, which differentiates all integrals obtained from functions in $X$, but a function can be found in $Y$ whose integral is not differentiated by the given basis? This problem is still far from its final solution. The first result related to this problem is due to Hayes [1, page 155], who showed that it is possible to distinguish the spaces $L^{p}$ and $L^{q}$ for $p \neq q$ by constructing an appropriate differential basis. Then, Stokolos [3, 4] showed that it is possible to distinguish Orlicz spaces by constructing an appropriate differential basis. Apparently, everything that has been achieved on the problem of distinguishing spaces with the help of differential bases is exhausted by these two results.

The following classical results are fundamental for the theory of differentiation of integrals in $\mathbb{R}^{n}: \mathbf{B}_{s}$ differentiates $L\left(\log ^{+} L\right)^{n-s}$ (the case $s=n$ is due to Lebesgue [5], the case $s=1$ to Jessen et al. [6], and the case of arbitrary $s$ to Zygmund [7]). In 1935, Saks [8] showed that $\mathbf{B}_{1}$ does not differentiate $o\left(L\left(\log ^{+} L\right)^{n-1}\right)$. It was proved later in [9] that $\mathbf{B}_{s}$ does not differentiate the space $o\left(L\left(\log ^{+} L\right)^{n-s}\right)$.

Let $Q_{0}$ be the unit cube in $\mathbb{R}^{n}$ with the usual Lebesgue measure. For $f: Q_{0} \rightarrow \mathbb{R}$, we will denote by $\lambda(f, \gamma)$ the distribution function of $f$ :

$$
\lambda(f, \gamma)=\mu(\{t:|f(t)|>\gamma\})
$$

its rearrangement in nonincreasing order will be denoted by $f^{*}$ :

$$
f^{*}(t)=\inf \{s>0: \lambda(f, s)<t\} .
$$

A Banach space $X$ consisting of measurable functions is said to be ideal [10] if, given $y \in X$, measurability of $x$ and $|x(t)| \leq$ $|y(t)|$ almost everywhere, it follows that $x \in X$ and $\|x \mid X\| \leq$ $\|y \mid X\|$. An ideal space is said to be symmetric [10] if for $x, y \in X$, given that the inequality $\lambda(x, \gamma) \leq \lambda(y, \gamma)$ holds for all $\gamma \in \mathbb{R}_{+}$, it follows that $\|x|X\|\leq\| y| X\|$. As usual we denote by $X^{\prime}$ the space of integral functionals on $X$ with norm

$$
\left\|g \mid X^{\prime}\right\|=\sup \left\{\int_{Q_{0}} g(t) x(t) d t:\|x \mid X\| \leq 1\right\} .
$$

For each symmetric space $X$, its fundamental function is defined by means of the identity

$$
\psi(X, t)=\{\|\chi(D) \mid X\|: \mu(D)=t\},
$$

where $\chi(D)$ denotes the characteristic function of a set $D$. This function is nondecreasing and $t^{-1} \psi(X, t)$ does not increase. The spaces $L^{p}$ of Lebesgue, $L_{h}$ of Orlicz, $M(\psi)$ of Marzinkiewicz, and $\Lambda(\psi)$ of Lorentz are the classical examples of symmetric spaces. The theory of symmetric spaces of functions is described in detail in [10] and that of symmetric spaces of sequences in [11]. Since Lorentz and Marzinkiewicz spaces will be used below, we will describe $M(\psi)$ and $\Lambda(\psi)$ in greater detail. Let us denote by $W$ the set of concave functions $\varphi:[0,1] \rightarrow \mathbb{R}_{+}$, each of which is continuous with $\varphi(0)=0$ and $\lim _{t \rightarrow 0} t^{-1} \varphi(t)=\infty$. Then, the
Lorentz space $\Lambda(\varphi)$ (resp., the Marzinkiewicz space $M(\varphi)$ ) consists of all those $f$ for which the following expression

$$
\|f \mid \Lambda(\varphi)\|=\int_{0}^{1} f^{*}(s) d \varphi(s)=\int_{0}^{\infty} \varphi(\lambda(f, s)) d s .
$$

Respectively,

$$
\begin{aligned}
\|f \mid M(\varphi)\| & =\sup _{0<t<1} \frac{1}{\varphi(t)} \int_{0}^{t} f^{*}(s) d s \\
& =\sup _{D \subset Q_{0}} \frac{1}{\varphi(\mu(D))} \int_{D}|f(s)| d s,
\end{aligned}
$$

is a finite norm. It is clear that for the fundamental functions of the Lebesgue, Orlicz, Lorentz, and Marzinkiewicz spaces we have the identities

$$
\begin{gathered}
\psi\left(L^{p}, t\right)=t^{1 / p}, \quad \psi\left(L_{h}, t\right)=\frac{1}{h^{-1}\left(t^{-1}\right)}, \\
\psi(\Lambda(\varphi), t)=\varphi(t), \quad \psi(M(\varphi), t)=\frac{t}{\varphi(t)} .
\end{gathered}
$$

The duality between Lorentz and Marzinkiewicz spaces,

$$
\Lambda(\varphi)^{\prime}=M(\varphi), \quad M(\varphi)^{\prime}=\Lambda(\varphi)
$$

is well known, as is the embedding theorem of Krejn et al. [10]: let $X$ be a symmetric space and $\psi(X, t)=\varphi(t)$ its fundamental function; then we have the continuous embeddings

$$
\Lambda(\varphi) \subseteq X \subseteq M\left(\frac{t}{\varphi(t)}\right)
$$

If $\varphi(t)=t^{\theta}$ with $0<\theta<1$, then in this case the Lorentz (Marzinkiewicz) space will be denoted by $\Lambda^{\theta}\left(M^{\theta}\right)$.

\section{The Construction of Differential Bases}

Now we pass to the construction of differential bases. For simplicity and clarity, we will demonstrate the construction for $n=2$. We begin the construction of the corresponding bases from the construction of a certain collection $U$ of sets in the square $Q_{0}=[0,1] \times[0,1]$; we will refer to the construction process as the basic construction.

Suppose that we are given a sequence of triples of natural numbers $\left(m_{i}, p_{i}, l_{i}\right)$, for which $m_{i+1}>m_{i}, p_{i+1}>p_{i}$, and $l_{i+1}>l_{i}$. First we divide the square $Q_{0}$ into $m_{1}^{2}$ equal squares $Q(1, i)\left(i=1,2, \ldots, m_{1}^{2}\right)$. These we will call squares of the first level. Then again we divide each of the squares $Q(1, i)$ into $m_{2}^{2}$ equal squares and obtain the squares $Q(2, i)(i=$ $\left.1,2, \ldots, m_{1}^{2} m_{2}^{2}\right)$. These we will call squares of the second level, and so on. Each of the squares of the $k$ th level $Q(k, i)$ is divided in turn into $p_{k} l_{k}$ equal rectangles $\Pi(k, i, j)\left(j=1,2, \ldots, p_{k} l_{k}\right)$, which we will take as open. For the sake of definiteness, we will agree that the vertical side of the square is divided into $p_{k}$ equal parts, while the horizontal side of the square is divided into $l_{k}$ equal parts. Now, we will construct a collection of sets $U(k)$ relating to the $k$ th level. 
We take the square of the $k$ th level with the first index. The sets of the collection $U(k)$ which are contained in the square $Q(k, 1)$ are formed in the following manner. By construction the square is split up into $l_{k}$ vertical strips and $p_{k}$ horizontal strips which form $p_{k} l_{k}$ equal rectangles. We take the lower left rectangle $\Pi(k, 1,1)$, which lies at the intersection of the first horizontal and the first vertical. All the other rectangles from the square $Q(k, 1)$ lying on the first horizontal do not take part in the continuation of the construction and can be discarded. The set $U(k, 1,1)$ consists of the rectangle $\Pi(k, 1,1)$ to which have been added the $p_{k}-1$ rectangles from the first vertical. The set $U(k, 1,2)$ consists of the rectangle $\Pi(k, 1,1)$ to which have been added the $p_{k}-1$ rectangles from the second vertical. Then, we again take the rectangle $\Pi(k, 1,1)$, adding to it the $p_{k}-1$ rectangles from the third vertical, and we will form the set $U(k, 1,3)$. We continue this process and thus obtain the collection of sets

$$
\left\{U(k, 1,1), \ldots, U\left(k, 1, l_{k}\right)\right\}=U(k, 1)
$$

The sets of the collection $U(k, 1)$ have three important properties:

$$
\begin{array}{r}
U(k, 1, j) \cap U(k, 1, l)=\Pi(k, 1,1) \quad(j \neq l), \\
\mu(U(k, 1, j))=p_{k} \mu(\Pi(k, 1,1))=\frac{\mu(Q(k, 1))}{l_{k}}, \\
\operatorname{diam}(U(k, 1, j+1)) \geq \operatorname{diam}(U(k, 1, j)) \\
\left(j=1, \ldots, l_{k}-1\right) .
\end{array}
$$

Now, we repeat this construction for all the remaining squares $Q(k, i)$ of the $k$ th level $\left(i=1,2, \ldots, m_{1}^{2} m_{2}^{2} \cdots m_{k}^{2}\right)$. We will obtain the collections of sets $U(k, i)$. The sets of the collections $U(k, i)$ have the following important properties:

$$
\begin{gathered}
U\left(k, i_{1}, j\right) \cap U\left(k, i_{2}, l\right)=\emptyset \quad\left(i_{1} \neq i_{2}\right), \\
U(k, i, j) \cap U(k, i, l)=\Pi(k, i, 1) \quad(j \neq l), \\
\mu(U(k, i, j))=p_{k} \mu(\Pi(k, 1,1))=p_{k} \mu(\Pi(k, i, 1)) \\
=\frac{\mu(Q(k, 1))}{l_{k}}, \\
\lim _{k \rightarrow \infty} \operatorname{diam}(U(k, i, j))=0 .
\end{gathered}
$$

We now define the collection $U(k)$ as the union over $i$ of all the collections $U(k, i)$. Now on combining the collections of sets $U(1), U(2), \ldots, U(i), \ldots$ constructed for each level, we obtain the desired collection $U$. We note that the measure of those points of $Q_{0}$ which are not included in the sets of the collection $U(k)$ does not exceed $p_{k}^{-1}$. Therefore, from the definition and (15), we may take $U$ as a differential BF basis.
Corresponding to the collection $U(k)$, we construct the maximal operator

$$
\begin{aligned}
M_{k} x(t)=\sup & \left\{\frac{1}{\mu(U(k, i, j))}\right. \\
& \left.\times \int_{U(k, i, j)}|x(t)| d t: t \in U(k, i, j) \in U(k)\right\},
\end{aligned}
$$

and for the collection $U$ and $\delta>0$, we construct its maximal operator

$$
\begin{aligned}
& M_{\delta} x(t) \\
& \quad=\sup \left\{\frac{1}{\mu(B)} \int_{B}|x(t)| d t: t \in B \in U, \operatorname{diam} B<\delta\right\} .
\end{aligned}
$$

For $\delta=\operatorname{diam} Q_{0}$, we will write simply $M$ in place of $M_{\delta}$. We denote by $\Gamma(U(k))$ the union of the boundaries of all the sets $U(k, i, j)$ appearing in the collection $U(k)$. It is easy to see that the identity $\mu(\Gamma(U(k)))=0$ holds.

\section{An Effective Bound for Maximal Operator}

Our immediate goal is to obtain an effective bound for the maximal operator. The following lemma can be obtained easily from well-known facts of the metric theory of functions and the identity $\mu(\Gamma(U(k)))=0$.

Lemma 1. Suppose that the collections of sets $U(1), \ldots, U(n)$ have been constructed relative to the triples of natural numbers $\left(m_{i}, p_{i}, l_{i}\right)(i=1, \ldots, n)$. Then, for any $\epsilon>0$, there exists $a$ number $N=N(\epsilon)$ such that for all $m>N(\epsilon)$ the following condition is satisfied: if the square $Q_{0}$ is divided into $m^{2}$ equal nonintersecting squares $\left\{Q_{i}\right\}$, then one has the inequality

$$
\sum_{1}^{m^{2}}\left\{\mu\left(Q_{i}\right): Q_{i} \cap\left(\bigcup_{1}^{n} \Gamma(U(k))\right) \neq \emptyset\right\}<\epsilon .
$$

In other words, the area of those squares which intersect the boundary of at least one set of the collections $U(1), \ldots, U(n)$ will be less than $\epsilon$.

Now from all the differential bases obtained by means of the basic construction we extract a certain subclass for which it is possible to verify its differential properties.

Definition 2. We will say that a sequence of triples of natural numbers $\left(m_{k}, p_{k}, l_{k}\right)$ is admissible if the conditions

$$
\begin{aligned}
& m_{k+1}>m_{k}, \quad p_{k+1}>p_{k}, \\
& l_{k+1}>l_{k}, \quad m_{k+1}>N(\mu(\Pi(k, 1,1))),
\end{aligned}
$$

are satisfied, where the number $N(\cdot)$ is taken from Lemma 1 for $\epsilon=\mu(\Pi(k, 1,1))$. 
Definition 3. We will say that a differential basis $U=\{\bigcup U$ $(k)\}$ is admissible if the families $\{U(k)\}$ have been constructed by means of the basic construction applied with an admissible sequence of triples.

The following lemma is a key tool for the characterization of differential properties of admissible bases.

Lemma 4. Let $x \in L^{1}$ with $x(t)>0$ almost everywhere, and let $U$ be an admissible differential basis. Suppose that $\alpha>0$ and $\delta>0$ are given and choose $k(\delta)$ such that

$$
\begin{aligned}
& \operatorname{diam}(\Pi(k(\delta), 1,1)) \leq \delta, \\
& \text { but } \operatorname{diam}(\Pi(k(\delta)-1,1,1))>\delta .
\end{aligned}
$$

Then, one has the following relation:

$$
\left\{t: M_{\delta} x(t)>\alpha\right\} \subset E_{0} \cup E_{1} \cup E_{2} \text {, }
$$

where

$$
\begin{aligned}
& \mu\left(E_{0}\right)<2 \alpha^{-1} \int_{E_{0}} x(t) d t, \\
& \mu\left(E_{1}\right)<\sum_{k \geq k(\delta)} \mu(\Pi(k, 1,1)),
\end{aligned}
$$

and the set $E_{2}$ consists of the union of nonintersecting sets $V_{k}$ with $k \geq k(\delta)$, each of which can be represented in the form

$$
V_{k}=\{\bigcup Q(k, i): i \in J(k)\}
$$

for some $J(k)$, and moreover for each square $Q(k, i) \in V_{k}$ the condition

$$
\mu(Q(k, i))<2 \alpha^{-1} l_{k} \int_{\Pi(k, i, 1)} x(t) d t
$$

is satisfied.

Proof. We will establish the lemma for $\delta=\operatorname{diam} Q_{0}$. We will indicate all the changes required for other $\delta$ at the end. Let

$$
\begin{aligned}
D_{1} & =D(x, \alpha)=\{t: M x(t)>\alpha\} \\
& =\left\{\bigcup U(k, i, j): \frac{1}{\mu(U(k, i, j))} \int_{U(k, i, j)} x(t) d t>\alpha\right\}, \\
& I_{1}=\left\{(k, i, j): U(k, i, j) \subset D_{1}\right\}, \\
& k(1)=\min \left\{k:(k, i, j) \in I_{1}\right\} .
\end{aligned}
$$

We introduce the set

$$
W_{1}=\left\{\bigcup U(k(1), i, j):(k(1), i, j) \in I_{1}\right\} .
$$

Then, as it is not difficult to verify, we have the identity

$$
W_{1}=\left\{t: M_{k(1)} x(t)>\alpha\right\} .
$$

Let us put (recall that (15) holds)

$$
\begin{gathered}
J(k(1))=\left\{i: \int_{\Pi(k(1), i, 1)} x(t) d t>\frac{\alpha}{2} \mu(U(k(1), i, 1))\right. \\
\left.=\frac{\alpha \mu(Q(k(1), i))}{2 l_{k(1)}}\right\},
\end{gathered}
$$

$$
W_{1+}=\{\bigcup Q(k(1), i): i \in J(k(1))\} \text {, }
$$$$
W_{1-}=W_{1} \backslash W_{1+} \text {. }
$$

If $U(k(1), i, j) \subset W_{1-}$, then from the inequality

$$
\begin{aligned}
& \int_{U(k(1), i, j) \backslash \Pi(k(1), i, 1)} x(t) d t \\
& \quad=\int_{U(k(1), i, j)} x(t) d t-\int_{\Pi(k(1), i, 1)} x(t) d t \\
& >\alpha \mu(U(k(1), i, j))-\frac{\alpha}{2} \mu(U(k(1), i, j)) \\
& \quad=\frac{\alpha}{2} \mu(U(k(1), i, j)),
\end{aligned}
$$

we obtain

$$
\mu(U(k(1), i, j))<\frac{2}{\alpha} \int_{U(k(1), i, j) \backslash \Pi(k(1), i, 1)} x(t) d t .
$$

Now the sets $U\left(k(1), i, j_{1}\right) \backslash \Pi(k(1), i, 1)$ and $U\left(k(1), i, j_{2}\right) \backslash$ $\Pi(k(1), i, 1)$ are disjoint for $j_{1} \neq j_{2}$ according to (15). Therefore, we have the relation

$$
\begin{aligned}
\mu\left(W_{1-}\right) & \leq \sum_{U(k(1), I, j) \subset W_{1-}} \mu(U(k(1), i, j)) \\
& \leq \sum_{U(k(1), i, j) \subset W_{1-}} \frac{2}{\alpha} \int_{U(k(1), i, j) \backslash \Pi(k(1), i, 1)} x(t) d t \\
& \leq \frac{2}{\alpha} \int_{W_{1-}} x(t) d t .
\end{aligned}
$$

Then, if $Q(k(1), i) \subset W_{1+}$, according to (15) and the construction, we will obtain

$$
\begin{aligned}
\mu(Q(k(1), i)) & =l_{k(1)} \mu(U(k(1), i, 1)) \\
& <\frac{2 l_{k(1)}}{\alpha} \int_{\Pi(k(1), i, 1)} x(t) d t,
\end{aligned}
$$

and for the set $W_{1+}$ we will have according to the construction

$$
\mu\left(W_{1+}\right)=\sum_{i \in J(k(1))} \mu(Q(k(1), i)) .
$$

Next, among the sets $\left\{U(k, i, j): U(k, i, j) \subset D_{1}, k>k(1)\right\}$ we reject those which lie entirely in $W_{1-} \cup W_{1+}$ and put

$$
\begin{gathered}
D_{2}=\left\{\bigcup U(k, i, j): U(k, i, j) \subset D_{1},\right. \\
\left.U(k, i, j) \cap\left(W_{1-} \cup W_{1+}\right) \neq U(k, i, j)\right\}, \\
I_{2}=\left\{(k, i, j): U(k, i, j) \subset D_{2}\right\}, \\
k(2)=\min \left\{k:(k, i, j) \in I_{2}\right\} .
\end{gathered}
$$


We divide the set $D_{2}$ into two parts:

$$
\begin{aligned}
& D_{2 \gamma}=\left\{\bigcup U(k(2), i, j) \subset D_{2}:\right. \\
&\left.U(k(2), i, j) \cap\left(W_{1-} \cup W_{1+}\right) \neq \emptyset\right\}, \\
& W_{2}=\left\{U(k(2), i, j) \subset D_{2}:\right. \\
&\left.U(k(2), i, j) \cap\left(W_{1-} \cup W_{1+}\right)=\emptyset\right\} .
\end{aligned}
$$

Let us denote by $\overline{M_{k(2)}}$ the operator, analogous to $M_{k(2)}$ from (16), but with the upper bound taken only over those sets $U(k(2), i, j)$ which are disjoint from $W_{1-} \cup W_{1+}$. Then, as is not difficult to verify, we have the identity

$$
\begin{aligned}
W_{2} & =\left\{\bigcup U(k(2), i, j):(k(2), i, j) \in I_{2}\right\} \\
& =\left\{t: \overline{M_{k(2)}} x(t)>\alpha\right\} .
\end{aligned}
$$

Therefore, we can put

$$
\begin{gathered}
J(k(2))=\left\{i: \int_{\Pi(k(2), i, 1)} x(t) d t>\frac{\alpha}{2} \mu(U(k(2), i, 1))\right. \\
\left.=\frac{\alpha \mu(Q(k(2), i))}{2 l_{k(2)}}\right\}, \\
W_{2+}=\{\bigcup Q(k(2), i): i \in J(k(2))\}, \quad W_{2-}=W_{2} \backslash W_{2+} \cdot
\end{gathered}
$$

If $U(k(2), i, j) \subset W_{2-}$, then analogous to (30) we obtain

$$
\mu(U(k(2), i, j))<\frac{2}{\alpha} \int_{U(k(2), i, j) \backslash \Pi(k(2), i, 1)} x(t) d t .
$$

Because the sets $U\left(k(2), i, j_{1}\right) \backslash \Pi(k(2), i, 1)$ and $U(k(2), i$, $\left.j_{2}\right) \backslash \Pi(k(2), i, 1)$ are disjoint for $j_{1} \neq j_{2}$, we have the relations

$$
\begin{aligned}
\mu\left(W_{2-}\right) & \leq \sum_{U(k(2), i, j) \subset W_{2-}} \mu(U(k(2), i, j)) \\
& \leq \sum_{U(k(2), i, j) \subset W_{2-}} \frac{2}{\alpha} \int_{U(k(2), i, j) \backslash \Pi(k(2), i, 1)} x(t) d t \\
& \leq \frac{2}{\alpha} \int_{W_{2-}} x(t) d t .
\end{aligned}
$$

And for the set $W_{2+}$, we have in analogy to $W_{1+}$ the following relations: if $Q(k(2), i) \subset W_{2+}$, then

$$
\begin{aligned}
\mu(Q(k(2), i)) & =l_{k(2)} \mu(U(k(2), i, 1)) \\
& <\frac{2 l_{k(2)}}{\alpha} \int_{\Pi(k(2), i, 1)} x(t) d t \\
\mu\left(W_{2+}\right)= & \sum_{i \in J(k(2))} \mu(Q(k(2), i)) .
\end{aligned}
$$

The set $D_{2 \gamma}$ is made up of those $U(k(2), i, j)$ which intersect the boundaries of the sets $U(k(1), i, j)$ contained in
$W_{1-} \cup W_{1+}$. Since the basis is admissible, we can write the bound

$$
\mu\left(D_{2 \gamma}\right)<\mu(\Pi(k(1), 1,1)) .
$$

Now we reject those among the sets $\{U(k, i, j): U(k$, $\left.i, j) \subset D_{2}, k>k(2)\right\}$, which lie entirely in $\left(W_{1-} \cup W_{1+}\right) \cup$ $\left(W_{2-} \cup W_{2+}\right)$, and put

$D_{3}$

$$
\begin{aligned}
= & \left\{\bigcup U(k, i, j): U(k, i, j) \subset D_{1},\right. \\
& \left.U(k, i, j) \cap\left(\left(W_{1-} \cup W_{1+}\right) \cup\left(W_{2-} \cup W_{2+}\right)\right) \neq U(k, i, j)\right\},
\end{aligned}
$$$$
I_{3}=\left\{(k, i, j): U(k, i, j) \subset D_{3}\right\} \text {, }
$$$$
k(3)=\min \left\{k:(k, i, j) \in I_{3}\right\} \text {. }
$$

We divide the set $D_{3}$ into two parts:

$$
\begin{aligned}
D_{3 \gamma}=\left\{U(k(3), i, j) \subset D_{3}:\right. & \left.U(k(3), i, j) \cap\left(\left(W_{1-} \cup W_{1+}\right) \cup\left(W_{2-} \cup W_{2+}\right)\right) \neq \emptyset\right\}, \\
W_{3}=\left\{U(k(3), i, j) \subset D_{3}:\right. & \left.U(k(3), i, j) \cap\left(\left(W_{1-} \cup W_{1+}\right) \cup\left(W_{2-} \cup W_{2+}\right)\right)=\emptyset\right\},
\end{aligned}
$$

construct the sets $W_{3-}, W_{3+}$ and the operator $\overline{M_{k(3)}}$, and write analogues of (39), (40), (41), and so on. We continue this process to infinity and obtain as a result the collections of sets

$$
\begin{aligned}
& W_{1-}, W_{2-}, \ldots, W_{k-}, \ldots \\
& W_{1+}, W_{2+}, \ldots, W_{k+}, \ldots \\
& D_{2 \gamma}, D_{3 \gamma}, \ldots, D_{k \gamma}, \ldots
\end{aligned}
$$

Moreover,

$$
\begin{gathered}
W_{i+} \cap W_{j+}=\emptyset, \quad W_{i-} \cap W_{j-}=\emptyset \quad(i \neq j), \\
W_{i-} \cap W_{j+}=\emptyset,
\end{gathered}
$$

and the following relations hold:

$$
\mu\left(W_{k-}\right) \leq 2 \alpha^{-1} \int_{W_{k-}} x(t) d t
$$

and if $Q(k(i), j) \subset W_{i+1}$, then

$$
\begin{aligned}
& \mu(Q(k(i), i))=l_{k(i)} \mu(U(k(i), j, 1)) \\
& <\frac{2 l_{k(i)}}{\alpha} \int_{\Pi(k(i), j, 1)} x(t) d t, \\
& \mu\left(W_{i+}\right)=\sum_{j \in J(k(i))} \mu(Q(k(i), j)), \\
& \mu\left(D_{i \gamma}\right)<\mu(\Pi(k(i-1), 1,1)) .
\end{aligned}
$$


To complete the proof, it is enough to put

$$
\begin{gathered}
E_{0}=\left\{\bigcup W_{i-}: i=1,2, \ldots\right\}, \quad E_{1}=\left\{\bigcup D_{i \gamma}: i=2,3, \ldots\right\}, \\
E_{2}=\left\{\bigcup W_{i+}: i=1,2, \ldots\right\}, \\
V_{k(i)}=\{\bigcup Q(k(i), j): j \in J(k(i))\} .
\end{gathered}
$$

Then by (47), all the relations (22) and (24) will be satisfied for $\delta=\operatorname{diam} Q_{0}$ and $k(\delta)=1$. But if $\delta<\operatorname{diam} Q_{0}$, then all the arguments remain in force, only $k(1) \geq k(\delta)$, and therefore (22) and (24) are always true. The lemma is proved.

It is immediately useful to note that for admissible differential bases $\mu\left(E_{1}\right) \rightarrow 0$ as $\delta \rightarrow 0$.

Lemma 4 has a series of important corollaries.

Corollary 5. Each admissible differential basis is a density basis; that is, it differentiates $L^{\infty}$.

Proof. Let $x(t)=\chi(A)$ and fix $\alpha>0$. We first choose $k_{0}$ such that the inequality $0.5 \alpha>p_{k}^{-1}$ holds for $k \geq k_{0}$. Then by (15), we have for such $k$ the inequality

$$
\frac{1}{\mu(U(k, i, j))} \int_{\Pi(k, i, 1)} \chi(A) d t \leq \frac{\mu(\Pi(k, i, 1))}{\mu(U(k, i, j))}=p_{k}^{-1}<0.5 \alpha .
$$

Therefore, if we put $\delta=0.5 \operatorname{diam} \Pi\left(k_{0}, 1,1\right)$, then it will follow from (49) the definition of $\delta$ and the definition of the sets $V_{k}$ that $E_{2}=\emptyset$, and, consequently,

$$
\mu\left(\left\{t: M_{\delta} \chi(A)>\alpha\right\}\right) \leq \sum_{i>k_{0}} \mu(\Pi(i, 1,1))+2 \alpha^{-1} \mu(A) .
$$

It follows from (50) that $\mu\left(\left\{t: M_{\delta} \chi(A)>\alpha\right\}\right) \rightarrow 0$ as $\delta \rightarrow 0$ and $\mu(A) \rightarrow 0$. According to the criterion given in Theorem 1.1 of the book [1], the last observation implies that $U$ is a density basis.

Corollary 6. Let $X=L^{p}(1<p<\infty)$. If the relation

$$
\sup _{i} \frac{l_{i}^{q-1}}{p_{i}}=C<\infty
$$

holds, where $1 / p+1 / q=1$, then $U$ differentiates $L^{p}$.

Proof. Let $x \in L^{p}$ with $x \geq 0$ almost everywhere. We will bound the measure of the set $\left\{t: M_{\delta} x(t)>\alpha\right\}$ in accordance with Lemma 4 . We obtain from duality and (22) for any symmetric space $X$ the following:

$$
\begin{aligned}
& \mu\left(E_{0}\right) \\
& \quad \leq \frac{2}{\alpha}\|x \mid X\| \min \left\{\psi\left(X^{\prime}, \mu\left(E_{0}\right)\right), \psi\left(X^{\prime}, \mu(\operatorname{supp} x)\right)\right\} .
\end{aligned}
$$

In the case $X=L^{p}$, we have

$$
\mu\left(E_{0}\right) \leq \frac{2}{\alpha}\left\|x \mid L^{p}\right\| \mu\left(E_{0}\right)^{1 / q} .
$$

To obtain the bound for $E_{2}$, we can put

$$
g(x, \delta, t)=\sum_{k \geq k(\delta)} \sum_{i \in J(k)} l_{k} \chi(\Pi(k, i, 1))
$$

and by (24), we can write the relation,

$$
\begin{aligned}
\mu\left(E_{2}\right) & \leq \sum_{k \geq k(\delta)} \sum_{i \in J(k)} \mu(Q(k, i)) \\
& \leq 2 \alpha^{-1} \sum_{k \geq k(\delta)} \sum_{i \in J(k)} l_{k} \int_{\Pi(k, i, 1)} x(s) \chi(\Pi(k, i, 1)) d s \\
& \leq 2 \alpha^{-1}\left\|x|X\|\| g(x, \delta, \cdot)| X^{\prime}\right\| .
\end{aligned}
$$

But in our case

$$
\begin{aligned}
\left\|g(x, \delta, \cdot) \mid L^{q}\right\| & =\left(\sum_{k \geq k(\delta)} \sum_{i \in J(k)} l_{k}^{q} \mu(\Pi(k, i, 1))\right)^{1 / q} \\
& =\left(\sum_{k \geq k(\delta)} \sum_{i \in J(k)} l_{k}^{q} \frac{\mu(Q(k, i))}{l_{k} p_{k}}\right)^{1 / q} .
\end{aligned}
$$

Taking into account (15), (51), and (55) along with the fact that $E_{2}$ consists of nonintersecting sets $V_{k}$, and consequently of squares $Q(k, i)$, we obtain

$$
\mu\left(E_{2}\right) \leq 2 C \alpha^{-1} \mu\left(E_{2}\right)^{1 / q}\left\|x \mid L^{q}\right\| .
$$

If we put

$$
x_{k}(t)= \begin{cases}x(t) & \text { for } x(t) \geq k, \\ 0 & \text { for } x(t)<k,\end{cases}
$$

and apply the bounds (53) and (57) to $x_{k}(t)$, then we will obtain $\mu\left(\left\{t: M_{\delta} x_{k}(t)>\alpha\right\}\right) \rightarrow 0$ as $\delta \rightarrow 0$ and $k \rightarrow$ $\infty$. For density bases, the last observation is equivalent by the criterion given in Theorem 2.2 of the book [1] to the differentiability of the integral $\int x(t) d t$. Since this result is true for any $x \in L^{p}, x \geq 0$ almost everywhere, it follows that $U$ differentiates $L^{p}$. The corollary is proved.

Corollary 7. Let $\varphi \in W$ and $X=M(\varphi)$. If the relations

$$
\sum_{i \geq 1} l_{i} \varphi\left(\frac{1}{p_{i} l_{i}}\right)<\infty, \quad \sum_{i=k}^{\infty} \frac{1}{p_{i} l_{i}} \leq \frac{C}{p_{k} l_{k}},
$$

are satisfied and $C$ does not depend on $k \in \mathbb{N}$, then $U$ differentiates $M(\varphi)$.

Proof. The proof of this corollary follows the scheme of the proof of Corollary 6 . Let $x \in M(\varphi)$ with $x(t) \geq 0$ almost everywhere. We find a bound for the measure of the set 
$\left\{t: M_{\delta} x(t)>\alpha\right\}$ by using Lemma 4 and (52) and (55). From (52) and duality, we obtain

$$
\begin{aligned}
\mu\left(E_{0}\right) & \leq 2 \alpha^{-1}\|x \mid M(\varphi)\| \psi(\Lambda(\varphi), \mu(\operatorname{supp} x)) \\
& =2 \alpha^{-1}\|x \mid M(\varphi)\| \varphi(\mu(\operatorname{supp} x)),
\end{aligned}
$$

and we will determine directly the norm of $g(x, \delta, t)$ in $\Lambda(\varphi)$. According to (59) for $\gamma>l_{n} \geq l_{k(\delta)}$, we have the inequality

$$
\begin{aligned}
\mu(\{t: g(x, \delta, t)>\gamma\}) & \leq \sum_{k>n} \sum_{i \in J(k)} \mu(\Pi(k, i, 1)) \\
& \leq \sum_{k>n} \frac{1}{p_{i} l_{i}} \leq \frac{C}{p_{n+1} l_{n+1}},
\end{aligned}
$$

and for $\gamma \leq l_{k(\delta)}$, there is the bound

$$
\begin{aligned}
\mu(\{t: g(x, \delta, t)>\gamma\}) & \leq \sum_{k \geq k(\delta)} \sum_{i \in J(k)} \mu(\Pi(k, i, 1)) \\
& \leq \sum_{k \geq k(\delta)} \frac{1}{p_{i} l_{i}} \leq \frac{C}{p_{k(\delta)} l_{k(\delta)}} .
\end{aligned}
$$

Therefore,

$$
\begin{aligned}
\| g & (x, \delta, t) \mid \Lambda(\varphi) \| \\
& =\int_{0}^{\infty} \varphi \lambda(g(x, \delta, \cdot), \gamma) d \gamma \\
& =\left\{\int_{0}^{l_{k(\delta)}}+\sum_{j=k(\delta)}^{\infty} \int_{l_{j}}^{l_{j+1}}\right\} \varphi \lambda(g(x, \delta, \cdot), \gamma) d \gamma \\
& \leq l_{k(\delta)} \varphi\left(\frac{C}{p_{k(\delta)} l_{k(\delta)}}\right)+\sum_{j \geq k(\delta)}\left(l_{j+1}-l_{j}\right) \varphi\left(\frac{C}{p_{j+1} l_{j+1}}\right) \\
& \leq C \sum_{j \geq k(\delta)} l_{j} \varphi\left(\frac{1}{p_{j} l_{j}}\right) .
\end{aligned}
$$

From this, we obtain

$$
\mu\left(E_{2}\right) \leq \frac{2 C}{\alpha}\|x \mid M(\varphi)\| \sum_{j \geq k(\delta)} l_{j} \varphi\left(\frac{1}{p_{j} l_{j}}\right) .
$$

The final part of the proof repeats word for word the proof of the corresponding part in Corollary 6 .

Corollary 8. Let $\varphi \in W$ and $X=\Lambda(\varphi)$. If the conditions

$$
\sup _{n} \frac{1}{p_{n} \varphi\left(1 / p_{n+1} l_{n+1}\right)}<\infty, \quad \sum_{i=n}^{\infty} p_{i}^{-1}<C p_{n}^{-1},
$$

are satisfied and $C$ does not depend on $n \in \mathbb{N}$, then $U$ differentiates $\Lambda(\varphi)$.
Proof. Let $x \in \Lambda(\varphi)$ with $x(t) \geq 0$ almost everywhere. Using Lemma 4 and (52), and (55) we find a bound for the measure of the set $\left\{t: M_{\delta} x(t)>\alpha\right\}$. From duality and (52), we obtain

$$
\begin{aligned}
\mu\left(E_{0}\right) & \leq 2 \alpha^{-1}\|x \mid \Lambda(\varphi)\| \psi\left(M(\varphi), \mu\left(E_{0}\right)\right) \\
& =2 \alpha^{-1}\|x \mid \Lambda(\varphi)\| \frac{\mu\left(E_{0}\right)}{\varphi\left(\mu\left(E_{0}\right)\right)},
\end{aligned}
$$

and we will arrive at a bound for the norm of the function $g(x, \delta, t)$ in $M(\varphi)$ by use of (61) and (62). If $C\left(p_{n+1} l_{n+1}\right)^{-1} \leq$ $t<C\left(p_{n} l_{n}\right)^{-1}$, then

$$
\begin{aligned}
& \int_{0}^{t} g^{*}(x, \delta, s) d s \\
& \quad \leq \sum_{j=n}^{\infty} \int_{C\left(p_{j+1} l_{j+1}\right)^{-1}}^{C\left(p_{j} l_{j}\right)^{-1}} g^{*}(x, \delta, s) d s \\
& \quad \leq \sum_{j=n}^{\infty} C\left(\frac{1}{p_{j} l_{j}}-\frac{1}{p_{j+1} l_{j+1}}\right) g^{*}\left(x, \delta, \frac{C}{p_{j+1} l_{j+1}}\right) .
\end{aligned}
$$

According to the definition,

$$
\begin{aligned}
& g^{*}\left(x, \delta, \frac{C}{p_{j+1} l_{j+1}}\right) \\
& \quad=\inf \left\{s>0: \lambda(g(x, \delta, \cdot), s)<\frac{C}{p_{j+1} l_{j+1}}\right\} .
\end{aligned}
$$

Therefore, from (61), (62), and (65), we obtain

$$
\begin{aligned}
\mu\left(\left\{s>0: g(x, \delta, s)>l_{j}\right\}\right) & \leq \sum_{i>j} \frac{1}{p_{i+1} l_{i+1}} \\
& \leq \frac{1}{l_{j+1}} \sum_{i>j} \frac{1}{p_{i}}<\frac{C}{p_{j+1} l_{j+1}},
\end{aligned}
$$

and, consequently,

$$
g^{*}\left(x, \delta, \frac{C}{p_{j+1} l_{j+1}}\right) \leq l_{j} .
$$
have

Therefore, for $C\left(p_{n+1} l_{n+1}\right)^{-1} \leq t<C\left(p_{n} l_{n}\right)^{-1}$, we will

$$
\begin{aligned}
\int_{0}^{t} g^{*}(x, \delta, s) d s & \leq \sum_{j=n}^{\infty} C\left(\frac{1}{p_{j} l_{j}}-\frac{1}{p_{j+1} l_{j+1}}\right) l_{j} \\
& \leq \sum_{j=n}^{\infty} C\left(\frac{1}{p_{j}}-\frac{l_{j}}{p_{j+1} l_{j+1}}\right) \\
& \leq C \sum_{j=n}^{\infty} \frac{1}{p_{j}} \leq \frac{C^{2}}{p_{n}}
\end{aligned}
$$

Therefore,

$$
\|g(x, \delta, t) \mid M(\varphi)\| \leq C_{1} \sup _{n}\left\{\frac{1}{p_{n} \varphi\left(1 / p_{n+1} l_{n+1}\right)}\right\} .
$$

The final part of the proof is similar to that in the proof of the preceding corollary. 


\section{The Admissible Differential Bases}

We now construct for each admissible differential basis $U$ a certain function which will play one of the key roles in the resolution of the problems formulated at the beginning of this section. Suppose that we are given a differential basis $U$ which has been constructed relative to an admissible sequence of triples $\left(m_{i}, p_{i}, l_{i}\right)$. Suppose that we are also given a sequence of numbers $\left\{d_{i}\right\}$. We define the function $f_{0}(t)$ by means of the identity

$$
f_{0}(t)=\sum_{k=1}^{\infty} d_{k} \chi\left(\bigcup_{j \geq 1} \Pi(k, j, 1)\right) .
$$

It turns out that with a suitable choice of numbers $\left\{d_{i}\right\}$ the basis $U$ does not differentiate $\int f_{0}$.

Lemma 9. Let $U$ be a differential basis constructed relative to $\left(m_{i}, p_{i}, l_{i}\right)$. Suppose that the following relation holds for the sequence $\left\{d_{i}\right\}$ :

$$
\varlimsup_{i \rightarrow \infty} d_{i} p_{i}^{-1}=C>0
$$

Then, the differential basis $U$ does not differentiate $\int f_{0}$.

Proof. Choose a positive integer $k$ and put $A_{k}=\bigcup_{j \geq 1} \Pi(k$, $j, 1)$. Then, in accordance with the construction and condition (15), we will have for any set $U(k, i, j)$

$$
\begin{aligned}
& \frac{1}{\mu(U(k, i, j))} \int_{U(k, i, j)} f_{0}(t) \chi\left(A_{k}\right) d t \\
& \quad \geq \frac{1}{\mu(U(k, i, j))} \int_{\Pi(k, i, 1)} d_{k} \chi(\Pi(k, i, 1)) d t \\
& \quad \geq d_{k} \frac{\mu(\Pi(k, i, 1))}{\mu(U(k, i, j))}=d_{k} p_{k}^{-1} .
\end{aligned}
$$

Therefore, from (15) and (74), we have the identity

$$
\begin{array}{r}
\varlimsup_{k \rightarrow \infty} \mu\left(\left\{t: M_{k} f_{0}(t) \chi\left(A_{k}\right)>0.5 C\right\}\right) \\
\geq \lim _{k \rightarrow \infty} \mu\left(\bigcup_{i, j \geq 1} U(k, i, j)\right)=1 .
\end{array}
$$

If we note that $\mu\left(A_{k}\right)=\left(p_{k} l_{k}\right)^{-1} \rightarrow 0$ as $k \rightarrow \infty$, we can deduce from Theorem 3.2.2 in [1] that the basis $U$ does not differentiate $\int f_{0}$. The lemma is proved.

Now, we will produce certain conditions on the function (73) which should guarantee its membership of certain spaces.

Lemma 10. Let $\left(m_{i}, p_{i}, l_{i}\right)$ be an admissible collection of triples and put

$$
\gamma_{j}=\sum_{i=1}^{j} d_{i}, \quad \beta_{j}=\sum_{i=j}^{\infty}\left(p_{i} l_{i}\right)^{-1}, \quad A_{j}=\sum_{i=j}^{\infty}\left(p_{i} l_{i}\right)^{-1} \gamma_{i} .
$$

Then, the following inequalities hold:

$$
\begin{gathered}
\left\|f_{0} \mid \Lambda(\varphi)\right\| \leq C+\sum_{i=1}^{\infty} \gamma_{i} \varphi\left(\beta_{i}\right), \\
\left\|f_{0} \mid M(\varphi)\right\| \leq C \sup _{j} \frac{A_{j}}{\phi\left(\beta_{j+1}\right)} .
\end{gathered}
$$

Proof. We have from the definition the inequality

$$
\lambda\left(f_{0}, \gamma_{j}\right) \leq \sum_{k=j+1}^{\infty} \mu\left(\bigcup_{i \geq 1} \Pi(k, i, 1)\right) \leq \sum_{k=j+1}^{\infty} \frac{1}{p_{k} l_{k}}=\beta_{j+1} .
$$

Therefore,

$$
\begin{aligned}
& \left\|f_{0} \mid \Lambda(\varphi)\right\| \\
& \quad=\int_{0}^{\infty} \varphi\left(\lambda\left(f_{0}, \gamma\right)\right) d \gamma \\
& \quad \leq\left\{\int_{0}^{\gamma_{1}}+\sum_{i=1}^{\infty} \int_{\gamma_{i}}^{\gamma_{i+1}}\right\} \varphi\left(\lambda\left(f_{0}, \gamma\right)\right) d \gamma \\
& \leq \gamma_{1} \varphi\left(\lambda\left(f_{0}, 0\right)\right)+\sum_{i=1}^{\infty}\left(\gamma_{i+1}-\gamma_{i}\right) \varphi\left(\lambda\left(f_{0}, \gamma_{i}\right)\right) \\
& \quad \leq \gamma_{1} \varphi\left(\lambda\left(f_{0}, 0\right)\right)+\sum_{i=1}^{\infty}\left(\gamma_{i+1}-\gamma_{i}\right) \varphi\left(\beta_{i+1}\right) \\
& \leq \gamma_{1} \varphi\left(\lambda\left(f_{0}, 0\right)\right)+\sum_{i=1}^{\infty} \gamma_{i+1} \varphi\left(\beta_{i+1}\right),
\end{aligned}
$$

which also establishes (78). Let us verify (79). If $\beta_{j+1} \leq t<\beta_{j}$, then

$$
\begin{aligned}
\int_{0}^{t} f_{0}^{*}(t) d t & \leq \sum_{i=j}^{\infty} \int_{\beta_{i+1}}^{\beta_{i}} f_{0}^{*}(t) d t \\
& \leq \sum_{i=j}^{\infty}\left(\beta_{i}-\beta_{i+1}\right) f_{0}^{*}\left(\beta_{i+1}\right) .
\end{aligned}
$$

According to the definition of $f^{*}$ and (80)

$$
f^{*}\left(\beta_{i+1}\right)=\inf \left\{s>0: \lambda\left(f_{0}, s\right)<\beta_{i+1}\right\} \leq \gamma_{i} .
$$

Therefore, from the monotonicity of the function $\varphi(t)$, we obtain

$$
\begin{aligned}
\left\|f_{0} \mid M(\varphi)\right\| & =\sup _{t} \frac{1}{\varphi(t)} \int_{0}^{t} f_{0}^{*}(s) d s \\
& \leq C \sup _{j} \frac{1}{\varphi\left(\beta_{j+1}\right)} \sum_{i=j}^{\infty}\left(\beta_{i}-\beta_{i+1}\right) \gamma_{i} \\
& =C \sup _{j} \frac{A_{j}}{\varphi\left(\beta_{j+1}\right)} .
\end{aligned}
$$

The lemma is proved. 
Now, we have everything ready for the proof of the following theorem. We note that the fundamental functions of the spaces involved in Theorem 11 are the same.

Theorem 11. Let $1<p<\infty$. Then, there exists an admissible differential basis $U$ which differentiates $L^{p}$ but not $M^{1-\theta}(p=$ $1 / \theta, 1 / p+1 / q=1)$.

Proof. We will construct an admissible sequence of triples $\left(m_{i}, p_{i}, l_{i}\right)$ in the following way. First, we put $p_{i}=2^{i}$ and choose a monotone increasing sequence of natural numbers $\left\{l_{i}\right\}$ such that for sufficiently large $i$ we have the inequality

$$
1 \leq \frac{l_{i}^{q-1}}{p_{i}}<2
$$

This is possible because of the inequality $1<p<\infty$. Then, we choose a sequence $\left\{m_{i}\right\}$ such that the sequence of triples $\left(m_{i}, p_{i}, l_{i}\right)$ is admissible. The possibility of such a choice of $\left\{m_{i}\right\}$ is also obvious. Now, we construct the differential basis $U$ corresponding to $\left(m_{i}, p_{i}, l_{i}\right)$. It follows from (85) and Corollary 6 that $U$ differentiates $L^{p}$. Let us show that $U$ does not differentiate the Marzinkiewicz space $M^{1-\theta}$. With the help of formula (73), we construct the function $f_{0}(t)$, putting $d_{i}=p_{i}$. It then follows from Lemma 9 that the basis $U$ does not differentiate $\int f_{0}$. We show that $f_{0} \in M^{1-\theta}$. For this, we make use of Lemma 10. From the definition of $p_{i}$ and (85), we obtain

$$
\gamma_{i}=p_{j+1}-2 \simeq p_{j}, \quad A_{j} \simeq \frac{1}{l_{j}}, \quad \beta_{j} \simeq \frac{1}{p_{j} l_{j}} .
$$

Therefore,

$$
\begin{aligned}
\left\|f_{0} \mid M(\varphi)\right\| & \leq C \sup _{j} \frac{A_{j}}{\beta_{j+1}^{1-\theta}} \leq C_{1} \sup _{j} \frac{\left(p_{j} l_{j}\right)^{1-\theta}}{l_{j}} \\
& \leq C_{2} \sup _{j}\left(p_{j} l_{j}^{1-q}\right)^{1-\theta}<\infty .
\end{aligned}
$$

Thus, by Lemma 10, $f_{0} \in M^{1-\theta}$ but $U$ does not differentiate $\int f_{0}$, and, consequently, $U$ does not differentiate the Marzinkiewicz space $M^{1-\theta}$. The theorem is proved.

Now, we show that with the help of the bases which have been set up it is possible to distinguish Lorentz and Marzinkiewicz spaces with exactly the same quasiexponential fundamental functions.

Theorem 12. Let $\varphi \in W$ and suppose that the following conditions are satisfied:

$$
\begin{aligned}
& \sup _{i \geq 1} \frac{\varphi\left(2^{-(i+1)}\right)}{\varphi\left(2^{-i}\right)}=q_{1}<1, \\
& \sup _{i \geq 1} \frac{\varphi\left(2^{-i}\right)}{\varphi\left(2^{-(i+1)}\right)}=q_{2}<2 .
\end{aligned}
$$

Then, there exists a differential basis which differentiates $\Lambda(\varphi)$ but does not differentiate $M(t / \varphi(t))$.
Proof. We define the sequences $\left\{a_{i}\right\}_{1}^{\infty},\left\{b_{i}\right\}_{1}^{\infty}$ by means of the equations $a_{i}=1 / \varphi\left(2^{-i}\right), b_{i}=2^{i} \varphi\left(2^{-i}\right)$ and put $p_{i}=\left[a_{i}\right], l_{i}=$ $\left[b_{i}\right], d_{i}=a_{i}([a]$ denotes the integer part of $a)$. Now, we choose a sequence $\left\{m_{i}\right\}$ such that the sequence of triples $\left(m_{i}, p_{i}, l_{i}\right)$ is admissible and construct the corresponding basis $U$. Then, in accordance with (88), we have the following relations:

$$
\begin{aligned}
\sum_{i=n}^{\infty} p_{i}^{-1} & \simeq \sum_{i=n}^{\infty} a_{i}^{-1} \\
& =\sum_{i=n}^{\infty} \varphi\left(2^{-i}\right) \simeq \frac{\varphi\left(2^{-n}\right)}{1-q_{1}} \simeq \frac{p_{n}^{-1}}{1-q_{1}} .
\end{aligned}
$$

Since $p_{n} l_{n} \simeq a_{n} b_{n} \simeq 2^{n}$, then

$$
\sup _{n} \frac{1}{p_{n} \varphi\left(1 / p_{n} l_{n}\right)} \simeq \sup _{n} \frac{1}{a_{n} \varphi\left(2^{-n}\right)} \leq C .
$$

We therefore obtain from this inequality and (89) by Corollary 8 that $U$ differentiates $\Lambda(\varphi)$. Let us verify that $U$ does not differentiate $M(t / \varphi(t))$. For this, we construct the function $f_{0}$ according to (73). Since $d_{i}=a_{i}$ and $p_{i}=\left[a_{i}\right]$, then by Lemma $9 U$ does not differentiate $\int f_{0}$. We show that $f_{0} \in M(t / \varphi(t))$, for which we make use of Lemma 10. Since $p_{n} l_{n} \simeq 2^{n}$, we have by (88) the following relations:

$$
\begin{gathered}
\gamma_{j}=\sum_{i=1}^{j} d_{i}=\sum_{i=1}^{j} a_{i}=\sum_{i=1}^{j} \frac{1}{\varphi\left(2^{-i}\right)} \leq \frac{1}{\varphi\left(2^{-j}\right)\left(1-q_{1}\right)}, \\
\beta_{j}=\sum_{i=j}^{\infty} \frac{1}{p_{i} l_{i}} \simeq \sum_{i=j}^{\infty} 2^{-i} \leq \frac{C_{1}}{p_{j} l_{j}} \\
A_{j}=\sum_{i=j}^{\infty} \frac{\gamma_{i}}{p_{i} l_{i}} \leq \frac{1}{1-q_{1}} \sum_{i=j}^{\infty} \frac{a_{i}}{p_{i} l_{i}} \leq \frac{C_{2}}{1-q_{1}} \sum_{i=j}^{\infty} \frac{1}{l_{j}} \\
\leq \frac{C_{3}}{1-q_{1}} \sum_{i=j}^{\infty} \frac{1}{b_{i}}=\frac{C_{4}}{1-q_{1}} \sum_{i=j}^{\infty} \frac{2^{-i}}{\varphi\left(2^{-i}\right)} \\
\leq \frac{C_{5}}{\left(1-q_{1}\right)\left(1-q_{2}\right) l_{j}} .
\end{gathered}
$$

We therefore obtain from Lemma 10 and the fact that $\varphi(t) / t$ does not increase that

$$
\begin{aligned}
\left\|f_{0} \mid M\left(\frac{t}{\varphi(t)}\right)\right\| & \leq C_{6} \sup _{j} A_{j} \frac{\varphi\left(\beta_{j+1}\right)}{\beta_{j+1}} \\
& \leq C_{7} \sup _{j} \frac{1}{l_{j}} \varphi\left(\frac{1}{p_{j+1} l_{j+1}}\right) p_{j+1} l_{j+1} \\
& \leq C_{8} \sup _{j} \varphi\left(\frac{1}{p_{j} l_{j}}\right) p_{j} \\
& \leq C_{9} \sup _{j} \varphi\left(2^{-j}\right) p_{j} \leq C_{10} .
\end{aligned}
$$

Thus, $f_{0} \in M(t / \varphi(t))$, and it follows from Lemma 9 that $U$ does not differentiate $\int f_{0}$. The theorem is proved. 
Now, we show that if there is a pair of symmetric spaces with nonequivalent fundamental functions, then there exists a differential basis which differentiates one but not the other. For this, we consider first the case of Lorentz and Marzinkiewicz spaces.

Theorem 13. Let $\phi, \psi \in W$, let $\Lambda(\psi)$ be the Lorentz space and let $M(t / \varphi(t))$ be the Marzinkiewicz space. Suppose that

$$
\lim _{t \rightarrow 0} \frac{\psi(t)}{\varphi(t)}=0
$$

Then, there exists a differential basis $U$ which differentiates $M(t / \varphi(t))$ but $\operatorname{not} \Lambda(\psi)$.

Proof. It follows from (93) that $\psi(t) \leq a(t) \varphi(t)$, and it can be assumed that $a(t) \downarrow$ (as $t \rightarrow 0_{+}$), $a(0)=0$ and $a(t)$ is continuous. We choose a sequence $\left\{\delta_{i}\right\}$ such that the conditions of the following two inequalities are satisfied:

$$
\psi\left(\delta_{i+1}\right)<0.25 \psi\left(\delta_{i}\right), \quad a\left(\delta_{i}\right) \leq 10^{-i},
$$

and put

$$
\begin{gathered}
a_{i}=\frac{1}{2^{i} \psi\left(\delta_{i}\right)}, \quad b_{i}=2^{i} \frac{\psi\left(\delta_{i}\right)}{\delta_{i}}, \quad p_{i}=\left[a_{i}\right]+1, \\
l_{i}=\left[b_{i}\right]+1, \quad d_{i}=a_{i} .
\end{gathered}
$$

Then from (94) and the concavity of $\psi$ follow the inequalities

$$
\begin{aligned}
& a_{i+1}=\frac{1}{2^{i+1} \psi\left(\delta_{i+1}\right)} \geq 2 \frac{1}{2^{i} \psi\left(\delta_{i}\right)}=2 a_{i}, \\
& b_{i+1}=2^{i+1} \frac{\psi\left(\delta_{i+1}\right)}{\delta_{i+1}} \geq 2 \times 2^{i} \frac{\psi\left(\delta_{i}\right)}{\delta_{i}}=2 b_{i} .
\end{aligned}
$$

Let us construct the function $f_{0}$ according to (73). We will verify that $f_{0} \in \Lambda(\psi)$. For this, we make use of Lemma 10. According to (96), we will have

$$
\begin{aligned}
& \gamma_{j}=\sum_{i=1}^{j} d_{i}=\sum_{i=1}^{j} a_{i} \simeq a_{j}, \\
& \beta_{j}=\sum_{i=j}^{\infty} \frac{1}{p_{i} l_{i}} \simeq \frac{1}{p_{j} l_{j}} \simeq \frac{1}{a_{j} b_{j}} \simeq \delta_{j} .
\end{aligned}
$$

Therefore,

$$
\begin{aligned}
\left\|f_{0} \mid \Lambda(\psi)\right\| & \leq C+\sum_{j=1}^{\infty} \gamma_{j} \psi\left(\beta_{j}\right) \leq C+\sum_{j=1}^{\infty} \gamma_{j} \psi\left(\delta_{j}\right) \\
& \leq C+\sum_{j=1}^{\infty} \frac{\psi\left(\delta_{j}\right)}{2^{j} \psi\left(\delta_{j}\right)}<\infty .
\end{aligned}
$$

Thus, $f_{0} \in \Lambda(\psi)$. Now, we choose a sequence $\left\{m_{i}\right\}$ in such a way that the sequence of triples $\left(m_{i}, p_{i}, l_{i}\right)$ is admissible. It follows from the definition of $p_{i}$ and $d_{i}$ in (95) that $U$ does not differentiate $\int f_{0}$. Therefore, according to (98), $U$ does not differentiate the space $\Lambda(\psi)$.

It remains to be shown that $U$ differentiates the Marzinkiewicz space $M(t / \varphi(t))$. According to the definitions (95) and (96),

$$
\sum_{i=k}^{\infty} \frac{1}{p_{i} l_{i}} \simeq \frac{1}{p_{k} l_{k}} \simeq \delta_{k} .
$$

On the other hand we obtain from the inequality $\psi(t) \leq$ $a(t) \varphi(t),(94)$, and (95), that

$$
\begin{aligned}
& \sum_{i=1}^{\infty} l_{i} \frac{1}{p_{i} l_{i}} \frac{1}{\varphi\left(1 / p_{i} l_{i}\right)} \\
& \leq C \sum_{i=1}^{\infty} \frac{1}{p_{i}} \frac{a\left(1 / p_{i} l_{i}\right)}{\psi\left(1 / p_{i} l_{i}\right)} \leq C_{1} \sum_{i=1}^{\infty} \frac{1}{p_{i}} \frac{a\left(\delta_{i}\right)}{\psi\left(\delta_{i}\right)} \\
& \quad \leq C_{2} \sum_{i=1}^{\infty} 2^{i} \frac{\psi\left(\delta_{i}\right) a\left(\delta_{i}\right)}{\psi\left(\delta_{i}\right)} \leq C_{3} \sum_{i=1}^{\infty} 2^{i} a\left(\delta_{i}\right)<\infty .
\end{aligned}
$$

Thus, condition (59) for Corollary 7 is satisfied, and so $U$ differentiates $M(t / \varphi(t))$. The theorem is proved.

This theorem has an important corollary.

Corollary 14. Let $X, Y$ be a pair of symmetric spaces and $\varphi(t), \psi(t)$ their fundamental functions. If the condition (93) is satisfied, then there exists a differential basis $U$ which differentiates the space $X$ but not the space $Y$.

Proof. We apply the embedding theorem (12) for the spaces $X$ and $Y$. Then,

$$
\Lambda(\varphi) \subseteq X \subseteq M\left(\frac{t}{\varphi(t)}\right), \quad \Lambda(\psi) \subseteq Y \subseteq M\left(\frac{t}{\psi(t)}\right) .
$$

Now, we apply Theorem 13 to the spaces $\Lambda(\psi)$ and $M(t / \varphi(t))$. As a result of this, we find a basis $U$ which differentiates $M(t / \varphi(t))$, and consequently $X$, but does not differentiate $\Lambda(\psi)$, and consequently $U$ does not differentiate $Y$.

\section{References}

[1] M. Gusman, Differentsirovanie of Integras in $\mathbb{R}^{n}$, Mir, Moscow, Russia, 1978, Translated from the English Edition: Lecture Notes in Mathematics No. 481, Berlin, Heidelberg, New York, Springer-Verlag (1975).

[2] E. I. Smirnov, Hausdorff Spectra in Functional Analysis, Springer, London, UK, 2002.

[3] A. M. Stokolos, "On the differentiation of integrals of functions from $L \varphi(L)$," Studia Mathematica, vol. 88, no. 2, pp. 103-120, 1988.

[4] A. M. Stokolos, "On the differentiation of integrals of functions from Orlicz classes," Studia Mathematica, vol. 94, no. 1, pp. 35$50,1989$.

[5] H. Lebesgue, "Sur l'intégration des fonctions discontinues," Annales Scientifiques de l'École Normale Supérieure, vol. 27, pp. 361-450, 1910. 
[6] B. Jessen, J. Marzinkiewicz, and A. Zygmund:, "A note on the differentiality of multiple integrals," Fundamenta Mathematicae, vol. 25, no. 1, pp. 217-234, 1935.

[7] A. Zygmund, "A note on the differentiability of integrals," Colloquium Mathematicum, vol. 16, pp. 199-204, 1967.

[8] S. Saks, "On the strong derivatives of functions of integrals," Fundamenta Mathematicae, vol. 25, pp. 235-252, 1935.

[9] B. López-Melero, "A negative result in differentiation theory," Studia Mathematica, vol. 72, no. 2, pp. 173-182, 1982.

[10] S. G. Krejn, I. Yu. Petunin, and E. M. Semenov:, Interpolation of Linear Operators, Nauka, Moscow, Russia, 1977.

[11] J. Lindenstrauss and L. Tzafriri, Classical Banach Spaces. I, II, Springer, Berlin, Germany, 1977. 


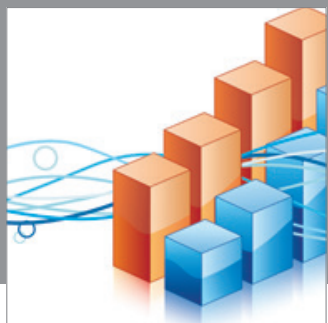

Advances in

Operations Research

mansans

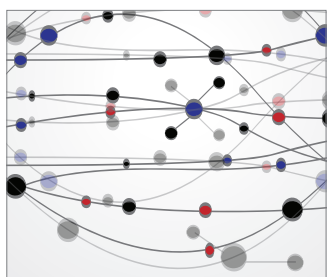

The Scientific World Journal
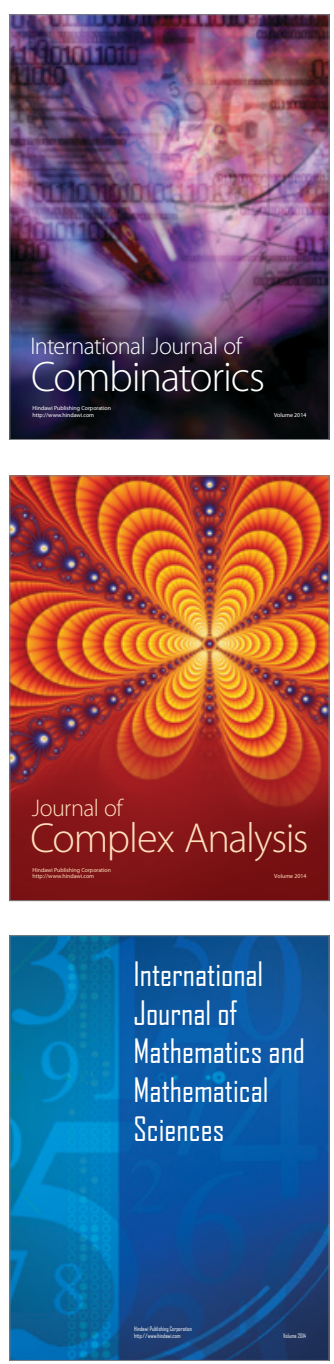
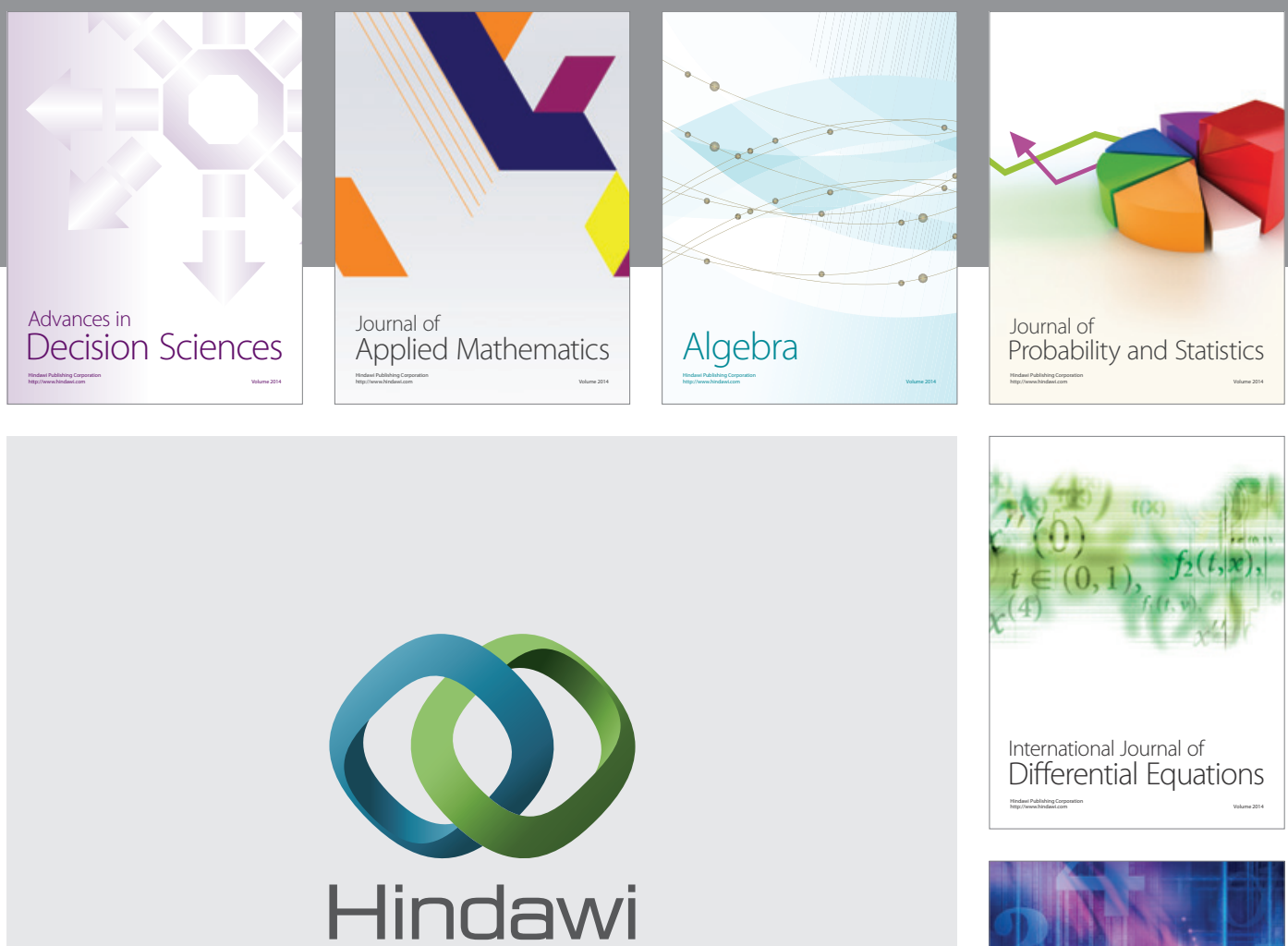

Submit your manuscripts at http://www.hindawi.com
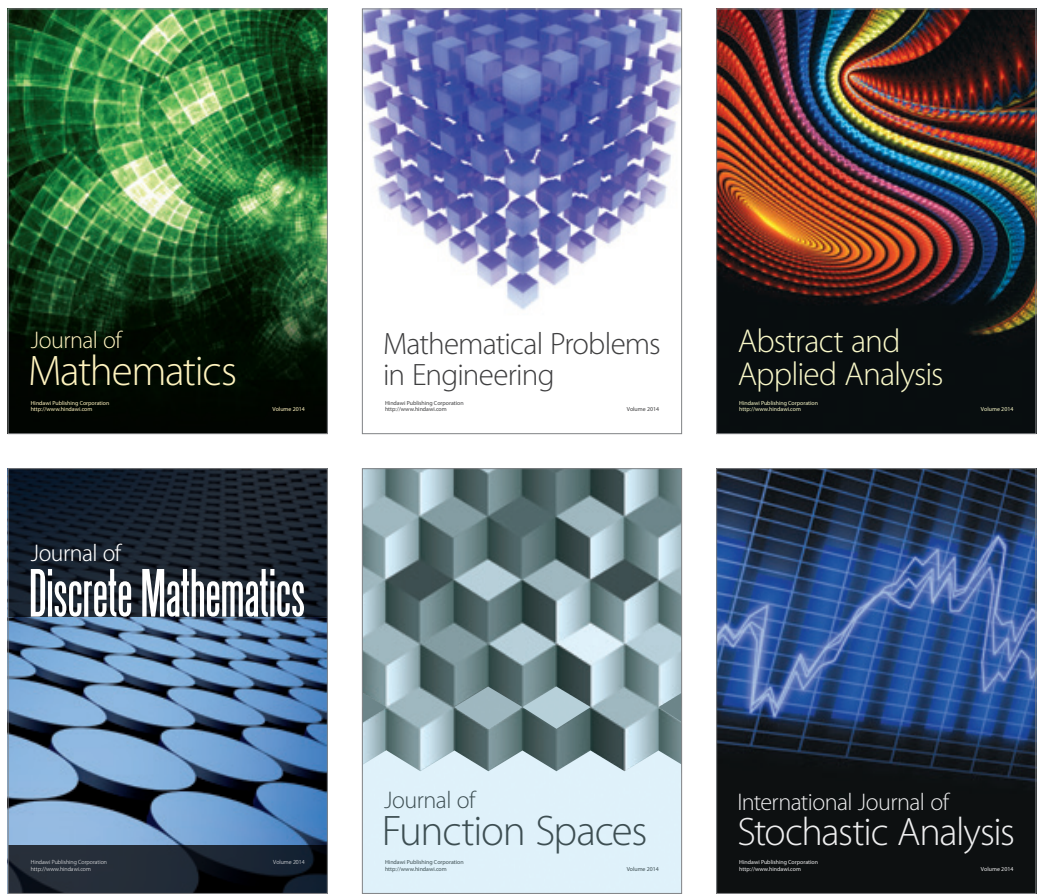

Journal of

Function Spaces

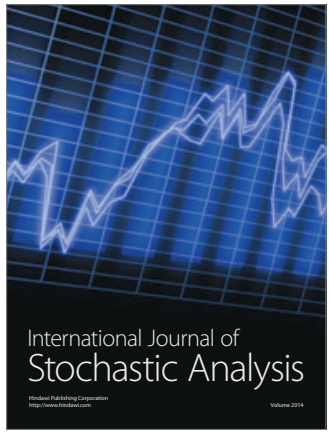

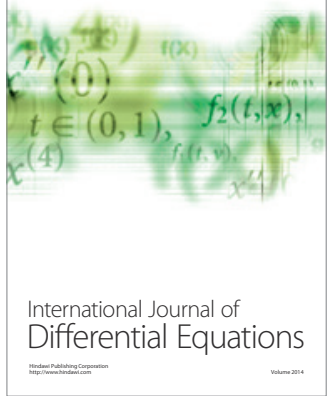
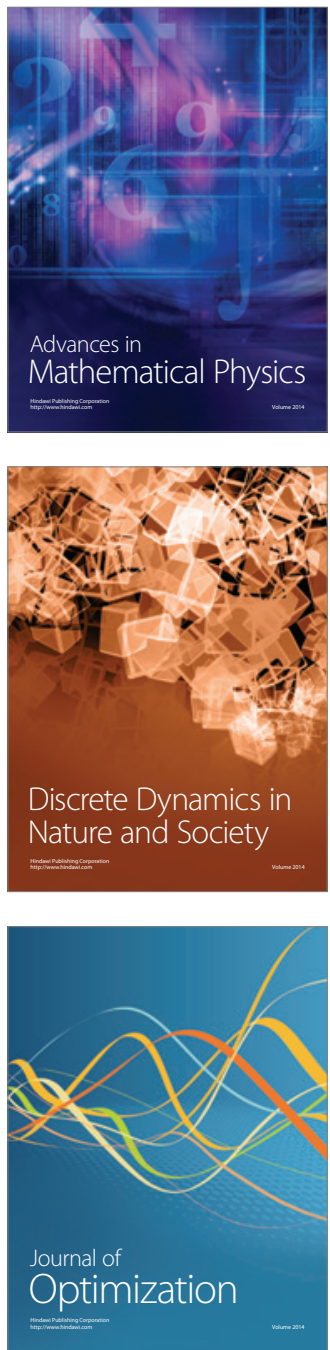